\title{
DUNKL-WILLIAMS TYPE INEQUALITIES FOR OPERATORS
}

\section{YOUYI JIANG AND LIMIN ZOU}

Abstract. The purpose of this paper is to discuss inequalities related to operator versions of the classical Dunkl-Williams inequality. We obtain refinements of some operator inequalities presented by Zou, He and Qaisar [Linear Algebra Appl. 438 (2013) 436-442].

Mathematics subject classification (2010): 47A63.

Keywords and phrases: Dunkl-Williams inequality, operator inequality, absolute value operator.

\section{REFERENCES}

[1] S. Abramovich, J. Barić, J. PeČarić, A new proof of an inequality of Bohr for Hilbert space operators, Linear Algebra Appl. 430 (2009), 1432-1435.

[2] F. Dadipour, M. FujiI, M. S. Moslehian, Dunkl-Williams inequality for operators associated with p-angular distance, Nihonkai Math. J. 21 (2010), 11-20.

[3] C. F. DunKL, K. S. Williams, A simple norm inequality, Amer. Math. Monthly. 71 (1964), 53-54.

[4] M. FuJII, H. Zuo, Matrix order in Bohr inequality for operators, Banach J. Math. Anal. 4 (2010), 21-27.

[5] M. FujiI, M. S. Moslehian, J. MićIĆ, Bohr's inequality revisited, Nonlinear analysis, Springer Optim. Appl. 68, 279-290, Springer, New York, 2012.

[6] J. PEČARIĆ, R. RAJIĆ, Inequalities of the Dunkl-Williams type for absolute value operators, J. Math. Inequal. 4 (2010), 1-10.

[7] K. -S. SAito, M. Tominaga, A Dunkl-Williams type inequality for absolute value operators, Linear Algebra Appl. 432 (2010), 3258-3264.

[8] F. ZhANG, On the Bohr inequality of operators, J. Math. Anal. Appl. 333 (2007), 1264-1271.

[9] L. Zou, C. HE, S. QAISAR, Inequalities for absolute value operators, Linear Algebra Appl. 438 (2013), 436-442.

[10] L. Zou, C. He, On operator Bohr type inequalities, Math. Inequal. Appl. 17 (2014), 1161-1169. 